\title{
DETC2006-99067
}

\section{EXTRACTION OF LOAD INFORMATION FROM PHOTOELASTIC IMAGES USING NEURAL NETWORKS}

\author{
Venketesh N Dubey, Gurtej S Grewal, Denzil J Claremont \\ Academic Biomedical Engineering Research Group \\ School of Design, Engineering \& Computing \\ Bournemouth University \\ Talbot Campus, Fern Barrow \\ Poole, BH12 5BB, UK \\ Tel. +44 1202965986 \\ Fax. +441202965314 \\ Email:vdubey@bmth.ac.uk
}

\begin{abstract}
Photoelastic materials develop colored fringes under white light when subjected to mechanical stresses which can be viewed through a polariscope. This technique has traditionally been used for stress analysis of loaded components, however, this can also be potentially used in sensing applications where the requirement may be measurement of the stimulating forces causing the generation of the fringes. This leads to inverse photoelastic problem where the developed image can be analyzed for the input forces. However, there could be infinite number of possible solutions which cannot be obtained by conventional techniques. This paper presents neural networks based approach to solve this problem. Experiments conducted to prove the principle have been verified with theoretical results and finite element analysis of the loaded specimens. The technique, if fully developed, can be implemented for any generalized case involving complex fringe patterns under different loading conditions for whole-field analysis of the stress pattern, which may find application in a variety of specialized areas including biomedical engineering and robotics.
\end{abstract}

Keywords: Fringe Information, Photoelasticity, Load Extraction, Neural Networks, Inverse problem.

\section{INTRODUCTION}

Photoelastic materials have unique property of exhibiting temporary birefringence when subjected to mechanical stresses. This produces colored fringe patterns in the material under white light, which can be visualized by suitable optical elements in the form of a polariscope. This characteristic is based on the phenomenon possessed by certain non-crystalline materials that are ideally isotropic but behave anisotropically when loaded [1]. The effect is temporary and persists only until specimen is loaded. This optical technique has been traditionally used for analyzing stress distribution in loaded models, however, this effect can be potentially used in sensing applications if the fringe information can be precisely quantified in terms of stress distribution and mapped onto the input force. This requires inverse problem to be solved i.e. finding the applied force information from the generated fringe patterns.

Photoelastic techniques like RGB calibration [2-4] and phase-shifting [5-7] have been used for stress-strain analysis but these cannot be directly used for retrieving the load/torque information. The reason being the model could have complex geometry and loading conditions, and the inverse may require prior parameter determination to define precise analytical relations for the problem. For such inverse problems, where it is difficult to develop analytical relations between information available and the output, neural networks approach may prove 
to be a useful tool, since neural networks are well suited to develop non-linear function between input and output [8-9]. It is also known that neural networks can tolerate slight experimental changes [10], which are likely to occur in real systems. In this paper we aim to prove the principle of using neural networks for the inverse photoelastic problem where direct image information from photoelastic fringes can be used to obtain the input function. The technique, if fully developed, can be implemented for any generalized case involving complex fringe patterns under different input conditions for whole-field analysis of the stress pattern, which may find application in a variety of specialized areas including robotics and biomedical engineering.

Some earlier researches have attempted the inverse photoelastic problems using neural networks, however, their technique relied on input from theoretical calculations; direct image data was not used [10]. In their work a tensile testing machine was used to apply bending load on a photoelastic beam and the fringe order was determined at known points. The stress difference was validated with an identical model using Finite Element Analysis (FEA). For the implementation of the neural network, entire training data were generated from the FEA to avoid errors in experimental data collection. In another work relevant to this research, a sensor was developed using photoelastic material and neural network to estimate torque from the optical intensity of the fringes [11], however, their intended application was different and the network training was inefficient. Photoelasticity has also been used in robotic applications for the development of force and slip sensors [12]. Cameron et al. [13] developed mathematical models to describe a slip sensor for edge detection. A similar model was developed by Eghtedari et al. [14] to study the effect of various design parameters like mechanical and optical properties of photoelastic model on the resolution of the slip sensor. This paper presents a neural network based approach which uses image information from the photoelastic fringes to determine the load information. The input given to the neural network is $\mathrm{R}$ intensity ${ }^{1}$ of the image and the output is verified with the applied load to the model. The presented technique is easy to implement, does not require extensive computation and can cope well within slight experimental variations. The technique just requires image acquisition, filtering and data extraction, which can then be fed to the neural network to provide load as the output. The novelty of this technique is that the neural network is trained with direct image data from actual experiments which requires no prior calculations. The network size is considerably small and system is generalized to analyze completely unseen data even at different camera settings. Also the technique dose not requires any knowledge of photoelasticity or FEA.

\section{METHODOLOGY}

The approach taken in this research is to establish direct association between the image information and the load applied to the model. For this, a circular disc was loaded under diametral compression with a known load and the developed fringes were recorded. The circular disc under diametral

\footnotetext{
${ }^{1} 8$-bit Red intensity curves across line of interest
}

compression is a common test model in photoelasticity for which analytical relations exist, which can be used for experimental verification. The acquired images will then be analyzed by two methods: (a) by estimating the fringe order and calculating the stress difference by the standard photoelastic relations [1] and (b) by conducting finite element analysis of the disc under diametral compression for the same load and thereby comparing the two analyses for the margin of error. The estimated stress difference can be put back into the analytical relation to find the input force and thus verifying this with the experimental data. This procedure will prove that force information is available which can be extracted from the photoelastic image within a certain tolerance band. However, this is an idealized case where loads are specified and analytical relations are available. For a general loading condition and geometry of the part such relations may not exist (as discussed later on) and some alternative approach needs to be considered to extract the load information.

In order to generalize the force estimation from a photoelastic image, a C-shaped model is considered for which direct analytical relations do not exist. The model is subjected to a bending load and the developed fringes are recorded. This model is then analyzed using FEA to compare one-to-one correspondence between the two stress images. From the image data it will be seen that identical force information are available but for such generalized cases, direct analytical relations are not available that can completely describe the whole stress field. Thus a neural network has been trained with the experimental fringe data to provide a model-free estimation of the applied force.

\section{IMAGE DATA AND THEORETICAL ANALYSIS}

Figure 1 shows fringes developed on a circular disc when a $95 \mathrm{~N}$ load is applied along its diameter. The photoelastic disc used in the experiment has an elasticity $(E)$ of $2500 \mathrm{~N} / \mathrm{mm}^{2}$, Poisson's ratio $(v)$ of 0.38 , strain optic co-efficient $(K)$ of 0.15 [15]. The disc has a diameter of $40 \mathrm{~mm}$ and thickness $(t)$ of $3.05 \mathrm{~mm}$. The band of fringes seen on the disc represents different stress zones which are designated by distinct fringe orders $(N)$. These fringe orders can be estimated from the color of the band and can be used to find the principal stress difference at the test points. In the shown image three test points are marked as points $1,2 \& 3$ with respect to the center of the disc. As a standard practice in photoelasticity, purple color band (tint of passage) is considered to have a fringe order of 1 and other distinct color bands have different fringe orders [16]. For the three marked points on the image the fringe orders are found to be close to $(1.22,1,0.6)$ respectively, as per the standard color identification scheme. In reflection photoelasticity the stress difference with reference to the above parameters can be given by equation (1):

$$
\left(\sigma_{1}-\sigma_{2}\right)=\frac{N}{2 t} \frac{E}{(1+v)} \frac{\lambda}{K}
$$

where, $\lambda$ is the wave length of white light usually taken as $5.77 \times 10^{-7} \mathrm{~m}$ [17]. This equation can be used to find stress 
difference at any point on the specimen as long as the fringe orders can be accurately estimated at those points. Table 1 gives the stress difference for each of these test points based the estimated fringe orders.

\begin{tabular}{|c|c|c|}
\hline Test points & Fringe order $(\boldsymbol{N})$ & Stress difference, $\mathbf{N} / \mathbf{m m}^{\mathbf{2}}$ \\
\hline 1 & 1.22 & 1.394 \\
\hline 2 & 1.0 & 1.142 \\
\hline 3 & 0.6 & 0.685 \\
\hline
\end{tabular}

Table 1. Stress difference calculated from the fringe order

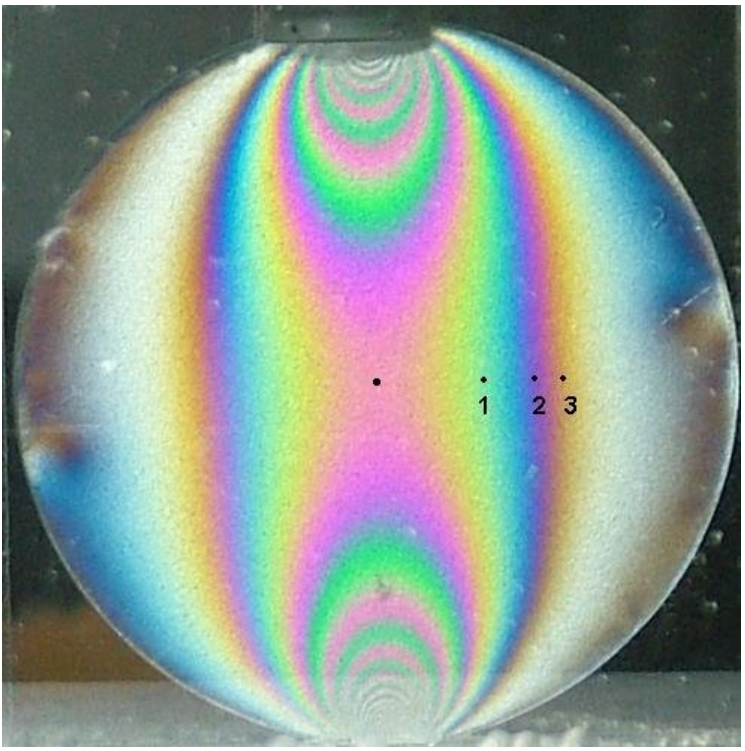

Figure 1. Image of a loaded photoelastic disc

The disc is then analyzed for same loading and boundary conditions using a FEA package. The result of the analysis is presented in Figure 2, which can be compared with the experimental model (Figure 1). From the stress pattern it can be clearly seen that there is a high degree of compatibility between the two results. The three test points are mapped from the experimental disc to the FEA model about its center (white dot) and the stress differences are estimated. The margin of error between the two analyses is shown in Table 2 .

\begin{tabular}{|c|c|c|c|}
\hline Test points & $\begin{array}{c}\text { Experimental stress } \\
\mathbf{N} / \mathbf{m m}^{\mathbf{2}}\end{array}$ & $\begin{array}{c}\text { FEA stress } \\
\mathbf{N} / \mathbf{m m}^{\mathbf{2}}\end{array}$ & \% error \\
\hline 1 & 1.394 & 1.339 & 3.9 \\
\hline 2 & 1.142 & 1.074 & 5.9 \\
\hline 3 & 0.685 & 0.710 & -3.6 \\
\hline
\end{tabular}

Table 2. Margin of error in experimental and FEA analyses

These errors are significantly high but this might have resulted from the manual mapping of points employed between the two images. This could, however, be minimized if the whole process could be automated. Nevertheless this validates the idea that stress information can be extracted from the photoelastic fringes which are in direct correspondence to the theoretical model.
The information of the fringe order can be further used to find the compressive load applied to the disc, which can be verified for each test points. Since the disc has well defined geometry and loading conditions analytical relation can be developed to find the applied load as given by equation (2):

$$
N=\frac{8 P R(1+v) K}{\pi \lambda E} \frac{R^{2}-\left(x^{2}+y^{2}\right)}{\left(x^{2}+y^{2}+R^{2}\right)^{2}-4 y^{2} R^{2}}
$$

where $P$ is the applied load, $R$ is the radius of the disc, and $x \&$ $y$ are the coordinates of the test points with respect to centre of the disc. It can be seen from Table 3 that for various test points $(1,2 \& 3)$ the calculated input load is close to the experimental load of $95 \mathrm{~N}$.

\begin{tabular}{|c|c|c|c|}
\hline Test points & Fringe order $(\boldsymbol{N})$ & Points $(\boldsymbol{x}, \boldsymbol{y})$ & Load (P), N \\
\hline 1 & 1.22 & $(6.40,0)$ & 89.03 \\
\hline 2 & 1.0 & $(8.93,0)$ & 98.33 \\
\hline 3 & 0.6 & $(11.87,0)$ & 92.11 \\
\hline
\end{tabular}

Table 3. Load data from the test points

This proves that for well-defined specimen geometry and boundary conditions the photoelastic image can be analyzed analytically to extract the load information. This procedure, however, may not be generalized and cannot be used directly for different loading conditions such as whole-field analysis of load data in biomedical sensing applications to assess the footprint of a diabetic patients or pressure patterns of disabled subjects as opposed to the discrete data available from strain gauge based sensors or load-cells.

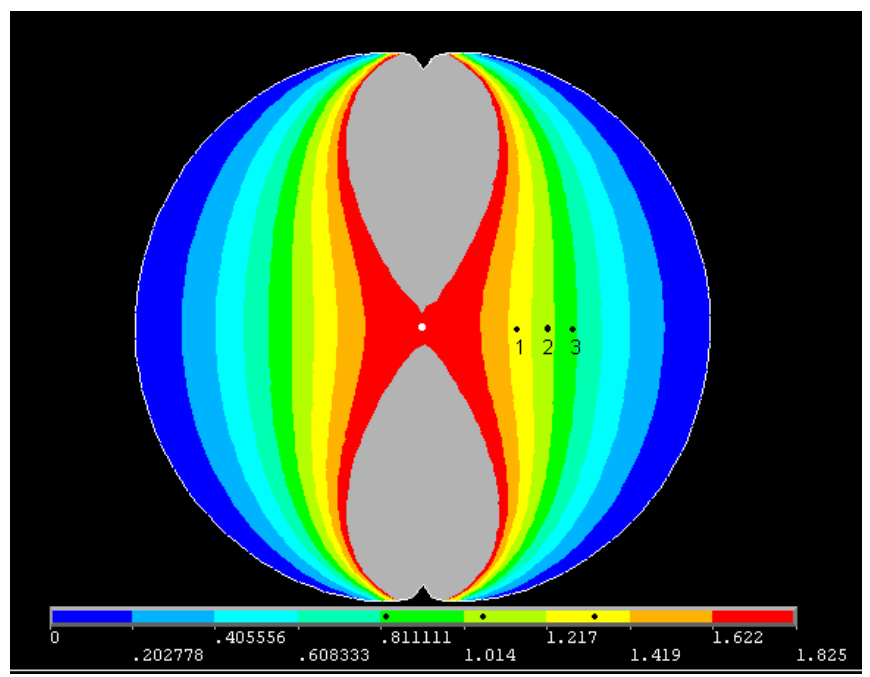

Figure 2. FEA of the disc under 95N diametral compression

To consider a generalized case a $\mathrm{C}$-shaped model was loaded as shown in Figure 3. This induced bending as well as axial load to the model. With the shown dimensions of the model an analytical relation can be developed for deflection $(\delta)$ at the tip of the shape with the load applied to the model. This is given by equation (3): 


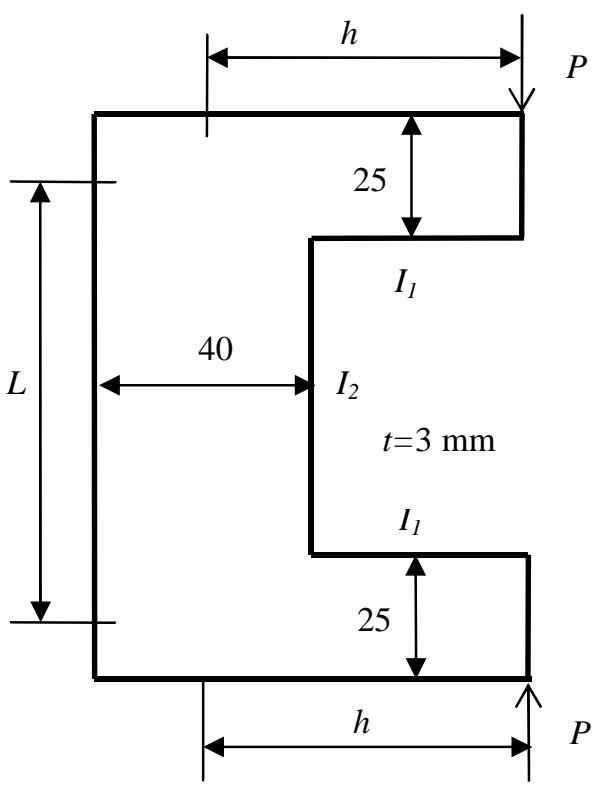

Figure 3. C-shaped model with dimensions (mm)

$$
\delta=\frac{P h^{2}}{3 E}\left[\frac{2 h}{I_{1}}+\frac{3 L}{I_{2}}\right]
$$

where $I_{i}$ is the second moment of area of the sections as shown. The stress pattern developed on the photoelastic model can be seen in Figure 4. The black fringe on the vertical arm of the section within the rectangular area, designated as the region of interest (ROI), represent the zone of zero stress lying on the neutral axis. The ROI contains linear and uniform stress distribution which is used for analyzing the fringe patterns. It is important to note, however, that similar fringe patterns can develop for a different set of loading conditions, which means that the inverse may have infinite number of solutions for the unknown input function. The image can be compared with the FEA of this model as shown in Figure 5.

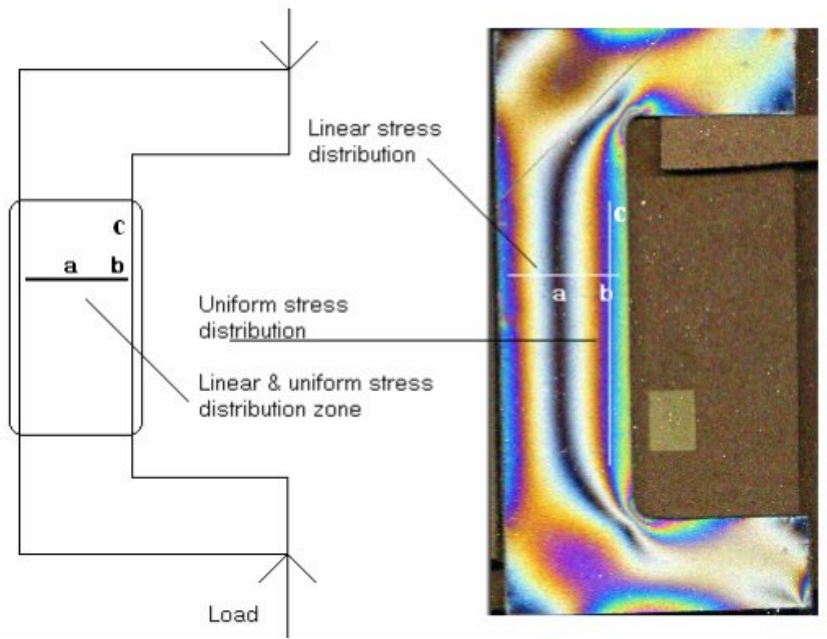

Figure 4: Model under bending load and the ROI
It can be seen that there is a high level of equivalence between the two stress patterns, however, due to irregular stress patterns throughout the section analytical relations cannot be developed that can completely define the whole stress field. It is therefore required to devise a system that can learn the input function from a large number of experimental images.

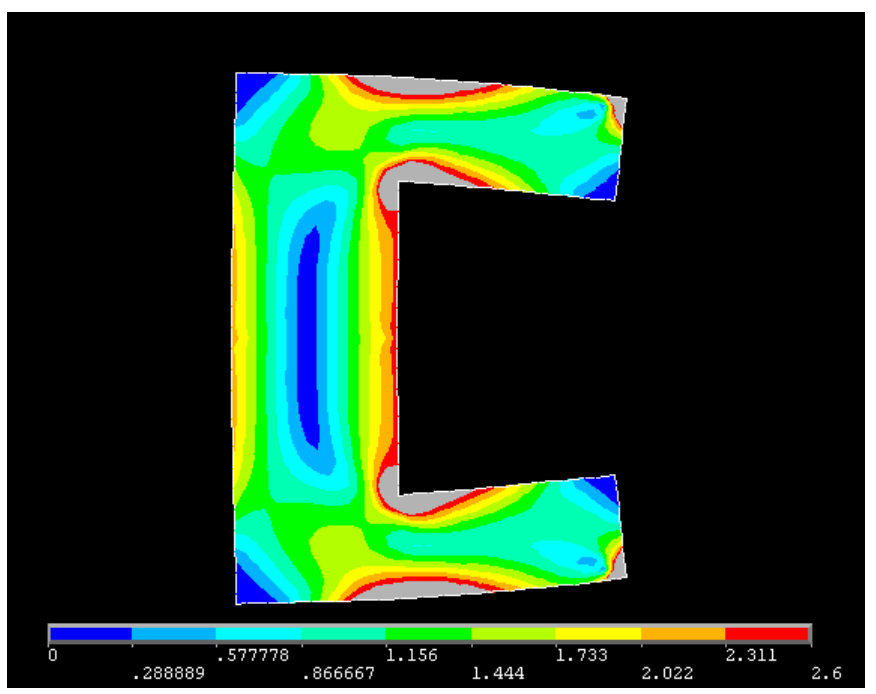

Figure 5. FEA of a C-shaped model with load at the tip

\section{NEURAL NETWORK BASED ANALYSIS}

As shown in Figure 4 the model was subjected to bending load and the fringe patterns were obtained from the ROI. The deflection was measured to one hundredth of a $\mathrm{mm}$ using a digital micrometer. This deflection was converted into the applied force using equation (3), which was set as the output target for the implemented network. The input to the neural network was given as R intensity of line ' $a-b$ ' as shown in the magnified view of the ROI (Figure 6). Considering the stress distribution along line 'c-b', it is evident that the stress is uniform. This facilitates extracting more than one input lines from the same image. There may be some noise introduced in the data if there is uneven loading at the two ends, however, it has been reported that noisy data can help in better generalization of the neural networks [18].

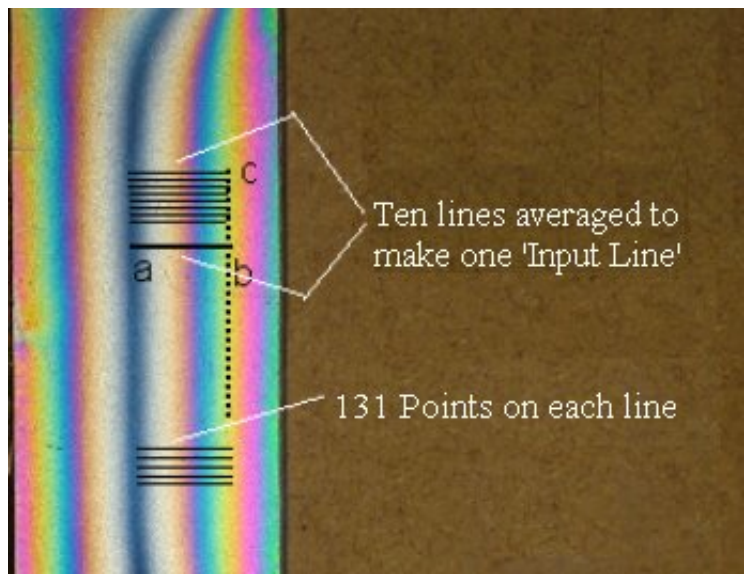

Figure 6: The ROI showing multiple lines for the input data 
The images were acquired at 1M-pixel resolution and filtered for data extraction before it was fed to the neural network. Image smoothing was performed to remove the irregularities present due to high sensitivity of the CCD chips. Average R-intensity of 10 input lines was extracted along 'cb' to make one input data line (131 points each) as shown in Figure 6. Similarly, 15 input data lines were extracted from each image under the load and the procedure was repeated for the successive loads. Figure 7 shows 15 input intensity curves obtained at a deflection of $0.4 \mathrm{~mm}$ applied to the model. The noise induced can be seen in the plot. The main consideration for selection of image data as direct input was to minimize the computational effort to obviate the problems of [10].

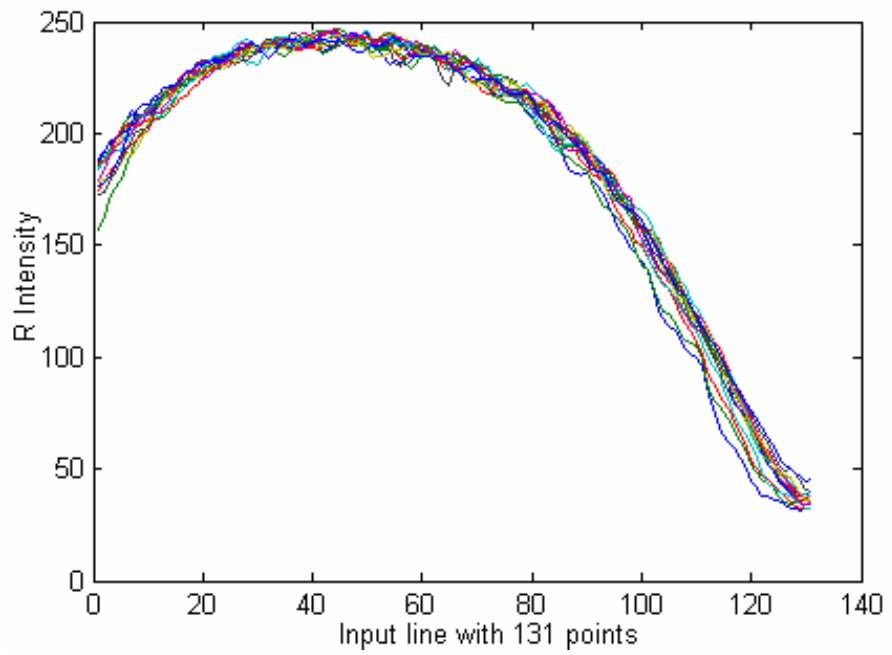

Figure 7: Band of noise in the input data

Ten similar sets of experiments were performed with 25 loading and unloading (13 different loads) allowing 15 input data to be extracted from each image at a particular deflection. Thus a total of 250 images were acquired. The deflection was incremented in steps of $0.08 \mathrm{~mm}$ from 0 to $0.96 \mathrm{~mm}$ using the micrometer screw. The neural network was trained with these sets of data to make it robust against slight changes that may happen during image acquisition e.g. changes in image orientation and scaling due to loading, lighting, reflections or camera settings. Conventionally, one load value would provide only one input data thus 250 input data sets from 250 images, but multi-input extraction gives 15 input sets from each image. This increase in training data from 250 to 3750 improves the robustness of the neural network.

As there are 131 points in each input line it is important to reduce dimensions of the input as it may lead to dimensionality curse [9], which in-turn increases the number of training patterns required. The Principal Component Analysis (PCA) [19] was used to reduce the input dimensionality. The input data was normalized between -1 to +1 since neural networks are found to be more efficient if data is presented in this range $[9,20]$.

A feed forward back propagation network was constructed as shown in Figure 8 with two hidden layers and training was done using 'Levenberg-Marquardt' approach [9]. The network trains by slowly modifying the weights and learns from the input data sets. The input data needs to be shuffled for efficient learning otherwise networks tend to memorize the last trained class of input data $[8,18]$.

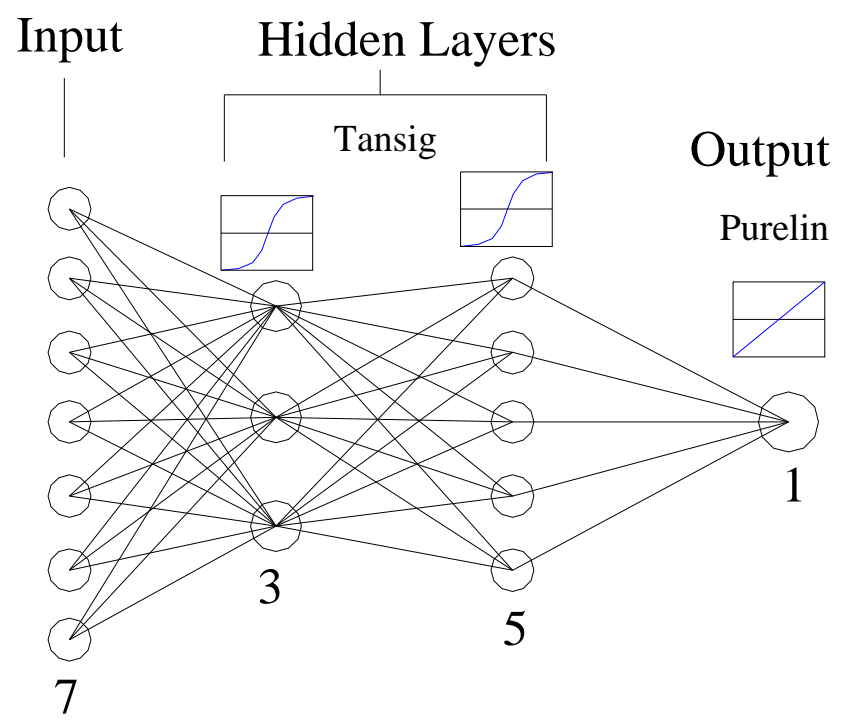

Figure 8: Structure of the neural network

In the implementation of the neural network the performance goal was based on mean square error (MSE) function. Weights were initialized randomly and the training was done with 50 different sets of initial weights. This is important since the error surface is unpredictable and by randomly initializing the weights can help in finding the global minima of the error surface otherwise the network may get stuck in local minima and will not generalize. The validation data (a subset extracted from total input data) was used to keep a check for generalization of the system and stop training in case of over-fitting (memorizing). The network size was kept considerably small since larger networks tend to memorize instead of learning [20].

\section{EXPERIMENTAL RESULTS}

In order to efficiently train the network the input dimensionality and the size of network was optimized. This was achieved by testing 49 different network size configurations for each input dimensionality and computing the error for 24 different test points. The best network size was found to be $\left\{\begin{array}{lll}7 & 3\end{array}\right\}$ which means that the final network had input dimensionality of 7 with 2 hidden layers, each with 3 and 5 neurons respectively (Fig. 8). The average percentage error for this network was found to be 4.2 and it further dropped to 2.78 when the initial loads were eliminated from the analysis since there was very little fringe information available at such low deflections. Once the optimal size of network was achieved and the network was trained, the weights were saved and the network is ready to accept the test data without any further training. 
The test results for unseen data were plotted in Figure 9. The plot between actual load and the load computed by the neural network can be seen almost at the same point in the figure. This shows that the system is capable of mapping output for the unseen data accurately.

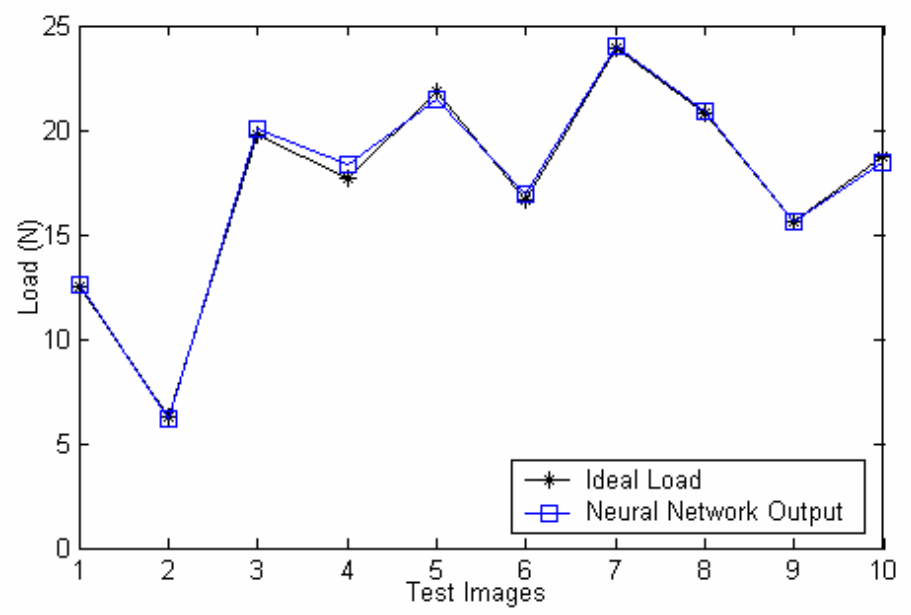

Figure 9: Neural network output with actual load curve

Regression analysis on the training data and the test results are shown in Table 4 . Under the ideal conditions of perfect fit (neural network output should equal the desired target) slope and the correlation coefficient should be 1 between the training and the test data.

\begin{tabular}{|l|c|c|}
\hline Data & Correlation coefficient & Slope \\
\hline Training & 0.993 & 0.985 \\
\hline Test & 0.999 & 0.985 \\
\hline
\end{tabular}

Table 4: Regression analysis on the training and the test data

It can be clearly seen from Fig. 9 and Table 4 that the actual load and the neural network output are very close to each other. It can be also concluded that the noise due to experimental variations during image acquisition do not have considerable effect on the system performance and it copes well within these variations.

\section{DISCUSSIONS AND CONCLUSIONS}

In this paper a new technique for extracting load information from photoelastic fringes has been proposed using neural networks. This technique will be particularly suitable for whole-field analysis of load data as opposed to discrete load information from strain gauge based sensors or the loadcells. If this technique could be fully developed as a sensing device, it could find application in many biomedical sensing areas such as early detection of diabetic foot ulceration or assessment of pressure sores in disabled subjects, thereby enacting prevention strategies by suitable footwear or bed designs. One of the main problems in assessment of diabetic foot ulceration is the difficulty of resolving the forces acting on the sole of the foot during standing and walking. The shear forces acting during walking is considered to be more harmful than the vertical force alone [21] as it occludes the blood flow more severely thus resulting in tissue dystrophy and rapid ulceration. However, the mechanism of ulceration due to biomechanical forces is not very well understood [22-23], partly because no sensors are available to resolve the forces and partly because these cannot be quantified. This research aims to tackle these problems by designing a sensing device and to interpret the data during actual condition of loading. A preliminary result from the newly designed sensing device based on the principles of photoelasticity is shown in Figure 10. The figure shows a portion of the foot-print of a healthy subject obtained during walking, but how to interpret and quantify this image for vertical and shear forces are engineering challenges. We are intending to implement neural network for this analysis and this paper is a first step in this direction.

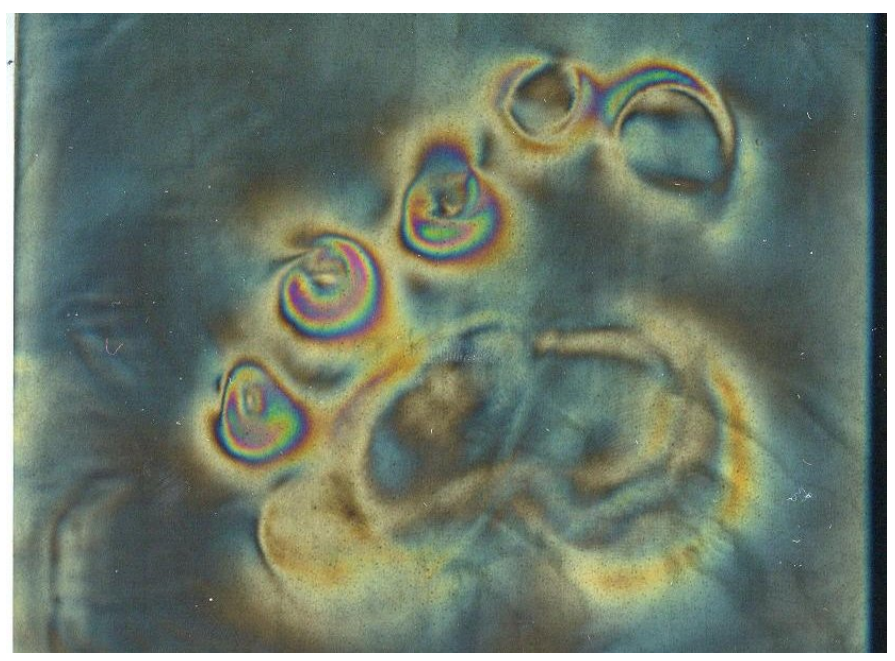

Figure 10: Foot-print of a healthy subject with the new sensor

The network presented in this paper was shown to be trained from direct image data without requiring any mathematical computation. It has also been shown that if sufficient and relevant inputs are provided, no prior calculations are needed for the training data and the results obtained were very close to the desired target. The output was found to be stable against slight experimental changes in loading conditions, lighting and camera settings. This leads to conclude that in absence of analytical solutions, neural network is a better approach for such inverse problems. The technique will be further developed for extracting load information for generalized and repeated loading conditions where the requirement may be whole-field image analysis of the photoelastic fringes in real time.

\section{ACKNOWLEDGMENTS}

This research was supported by a bursary awarded by the School of Design, Engineering \& Computing, Bournemouth University. 


\section{REFERENCES}

[1] Ramesh, K., 2000 "Digital photoelasticity: Advanced techniques and applications" Springer.

[2] Ajovalasit, A., Barone, S. and Petrucci, G., 1995 "Towards RGB Photoelasticity: Full-Field Started Photoelasticity in White Light" Experimental Mechanics, 35, 193-200.

[3] Quiroga, J., Botella, A. and Gomez-Pedrero, J., 2002 "Improved method for isochromatic demodulation by RGB calibration" Applied Optics, 41(17), 3461-3468.

[4] Ramesh, K. and Deshmukh, S., 1996 "Three fringe photoelasticity -use of colour image processing hardware to automate ordering of isochromatics" Strain, 32(3), 79-86.

[5] Patterson, E., 2002 "Digital Photoelasticity: Principles, Practice and Potential" Strain, 38, 27-39.

[6] Patterson, E., Hobbs, J. and Greene, R 2003 "A novel instrument for Transient Photoelasticity" Society for Experimental Mechanics, 43(4), 403-409.

[7] Asundi, A., 2002 "MATLAB for photomechanics: a primer" Elsevier.

[8] Patterson, D., 1996 "Artificial neural networks: Theory and applications" Prentice Hall,.

[9] Bishop, C., 1995 "Neural networks for pattern recognition" Oxford University Press.

[10] Noroozi, S., Amali, R. and Vinney, J. 2003 "Inverse problem approach using Photoelastic analysis and artificial neural networks in tandem" Strain, 40(2), 73-77.

[11] Chung, D., 1998 "Neural net based torque sensor using birefringent materials" Sensors and actuators A: Physical, 70(3), 243-249.

[12] Dubey, V. N. and Crowder, R. M., 2005 "Photoelasticity based dynamic tactile sensor", ASME International Design Engineering Technical Conference, DETC2005-84362, Long Beach, California (USA), Sept. 24-28.

[13] Cameron, A., Danial, R. and Durrant-White, H., 1988 "Touch and Motion" Proceedings IEEE international conference Robotics and Automation, 1-3, 1062-1067.

[14] Eghtedari., F., Hopkins, S. and Pham, D., 1993 "Model of a slip sensor" Proceeding international Mechanical Engineers, 207, 55-64.

[15] Photoelastic Materials, Bulletin S-116-G, 1992, Vishay Measurement Groups.

[16] Introduction to stress analysis by the Photostress method, Tech Note, TN-702-1, 1989, Vishay Measurement Groups.

[17] Zandman, F., et al., 1977 "Photoelastic coatings" Society for experimental stress analysis (SESA) monograph.

[18] Freeman, J. and Skapura, D., 1992 "Neural Networks: Algorithms, applications and programming techniques" Addison-Wesley.
[19] Bharath, R. and Drosen, J., 1994, "Neural networks and statistical analysis" Neural Network Computing, McGrawHill.

[20] MatLAB Manual, Mathworks, 2002.

[21] Davis, B., 1993, "Foot ulceration: hypothesis concerning shear and vertical forces acting on adjacent regions of the skin" Medical Hypothesis, 40:44-7.

[22] Cavanagh, P. et al., 2000 "New developments in the biomechanics of the diabetic foot" Diabetes Metab. Res. Rev 16(Sl)1:S6-S10.

[23] Curryer, $M$ and. Lemaire, E., 2000 "Effectiveness of various materials in reducing plantar shear forces: a pilot study" J. Am Podiatr Med. Assoc. 90(7): 346-53. 